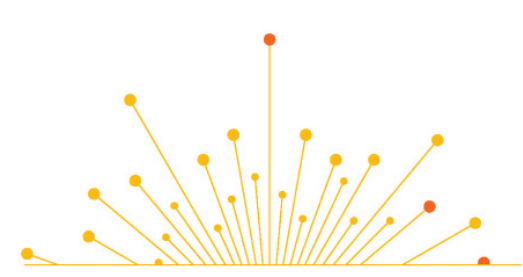

SCIENCE TABLE

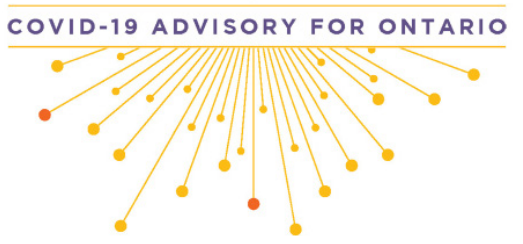

Version: 1.1

Published: September 30, 2021

Updated on October 4, 2021. Version 1.0 is available under Additional Resources at https://doi.org/10.47326/ocsat.2021.02.45.1.0

Citation: Bailey JJ, Morris AM, Bean S, et al. Evidence-based recommendations on the use of casirivimab + imdevimab, and sotrovimab for adults in Ontario. Science Briefs of the Ontario COVID-19 Science Advisory Table. 2021;2(45). https://doi.org/10.47326/ocsat.2021.02.45.1.0

Author Affiliations: The affiliations of the members of the Ontario COVID-19 Science Advisory Table can be found at https:// covid19-sciencetable.ca/.

Declarations of Interest: The declarations of interest of the members of the Ontario COVID-19 Science Advisory Table, its Working Groups, or its partners can be found at https:// covid19-sciencetable.ca/. The declarations of interest of external authors can be found under additional resources at https://doi. org/10.47326/ocsat.2021.02.45.1.0

About Us: The Ontario COVID-19 Science Advisory Table is a group of scientific experts and health system leaders who evaluate and report on emerging evidence relevant to the COVID-19 pandemic, to inform Ontario's response. Our mandate is to provide weekly summaries of relevant scientific evidence for the COVID-19 Health Coordination Table of the Province of Ontario, integrating information from existing scientific tables, Ontario's universities and agencies, and the best global evidence. The Science Table summarizes its findings for the Health Coordination Table and the public in Science Briefs.

The Drugs \& Biologics Clinical Practice Guidelines Working Group is a group of clinicians and scientists with recognized expertise in drugs, biologics, and clinical care. The Working Group evaluates existing scientific data, disease epidemiology, drug availability, and implementation issues in order to develop Clinical Practice Guidelines for the treatment of COVID-19 using drugs and biologics. The Working Group reports its findings to the public and the Science Table. Its findings are also summarized in Science

\title{
Evidence-Based Recommendations on the Use of Casirivimab + Imdevimab, and Sotrovimab for Adults in Ontario
}

Jacob J. Bailey, Andrew M. Morris, Sally Bean, Eyal Cohen, Jonathan Gubbay, Yona Lunsky, Samir Patel, Vanessa Tran, Katherine J. Miller, Zainab Abdurrahman, Stephanie Carlin, William Ciccotelli, Jennifer Gibson, Tiffany Kan, Bradley Langford, Elizabeth Leung, Ullanda Neil, Sumit Raybardan, Janet Smylie, Anupma Wadhwa, Peter Jüni, Menaka Pai, on behalf of the Ontario COVID-19 Science Advisory Table and the Drugs \& Biologics Clinical Practice Guidelines Working Group

\section{Key Message}

\section{Critically and Moderately III Patients}

In clinically unstable patients with no history of COVID-19 infection or full vaccination, casirivimab + imdevimab at a dose of $8000 \mathrm{mg}$ IV is recommended if patients are within 9 days of onset of any COVID-19 symptom AND have demonstrated rapid clinical deterioration. Antibody testing is not required in this case.

In clinically stable patients with or without a history of COVID-19 infection or full vaccination, casirivimab + imdevimab at a dose of $8000 \mathrm{mg}$ IV may be considered if patients are within 9 days of onset of any COVID-19 symptom AND are not at risk of acute decompensation AND if COVID-19 anti-spike antibody testing demonstrates that they are seronegative. Casirivimab + imdevimab is not recommended for moderately/ critically ill patients who are beyond 9 days of onset of any COVID-19 symptom, whether or not they are presumed to have immunity to SARS-CoV-2.

\section{Mildly III Patients}

Antibody testing is not required in mildly ill patients. In patients with no history of COVID-19 infection or full vaccination, casirivimab + imdevimab at a dose of $1200 \mathrm{mg}$ intravenous (IV) or subcutaneous (SC) is recommended if patients have confirmed, symptomatic COVID-19 AND are within 7 days of onset of any COVID-19 symptom AND have at least one of the following risk factors: age $>50$, obesity, cardiovascular disease (including hypertension), chronic lung disease (including asthma), chronic metabolic disease (including diabetes), chronic kidney disease, chronic liver disease, immunosuppression, or receipt of immunosuppressants.

In patients with a history of COVID-19 infection or full vaccination, casirivimab + imdevimab at a dose of $1200 \mathrm{mg}$ IV or SC may be considered if patients have confirmed symptomatic COVID-19 AND are within 7 days of onset of any COVID-19 symptom. In patients with a history of COVID-19 infection or full vaccination with risk factors other than immunocompromise or immunosuppression, SARS-CoV-2 neutralizing antibodies are not recommended as these patients have presumed immunity. In patients with no risk factors, SARS-CoV-2 neutralizing antibodies are not recommended as these patients are at low risk of adverse outcomes.

At this time, for mildly ill patients, casirivimab + imdevimab 1200mg IV or SC is preferred over sotrovimab 500mg IV due to practical considerations (i.e. it can also be administered subcutaneously).

Ontario's supply of SARS-CoV-2 neutralizing antibodies is limited, and demand by eligible patients may exceed supply in the near future. Understanding the impact of these agents on patient and system important outcomes will ensure that they are 
Briefs.

Correspondence to: Secretariat of the Ontario COVID-19 Science Advisory Table (info@covid19-sciencetable.ca)

Copyright: 2021 Ontario COVID-19 Science Advisory Table. This is an open access document distributed under the terms of the Creative Commons Attribution License, which permits unrestricted use, distribution, and reproduction in any medium, provided that the original work is properly cited.

The views and findings expressed in this Science Brief are those of the authors and do not necessarily reflect the views of all of the members of the Ontario COVID-19 Science Advisory Table, its Working Groups, and its partners. used to greatest benefit.

There are clear barriers to allocating SARS-CoV-2 neutralizing antibodies ethically and equitably. A number of strategies are suggested to address these barriers, including optimization of distribution, supply, administration, and allocation, dashboarding, and application of an evidence-informed risk framework for patient selection.

\section{Lay Summary}

When viruses infect you, your body mounts an immune response that includes the creation of protective molecules called antibodies. Antibodies can recognize very specific targets, such as specific proteins on the surface of a virus. People with antibodies to a virus can neutralize that same virus by recognizing it, attaching to it, and preventing it from spreading throughout the body and causing an infection.

\section{How SARS-CoV-2 Neutralizing Antibodies Work}

One of the proteins on the surface of the SARS-CoV-2 virus - the organism that causes COVID-19 - is the spike protein. SARS-CoV-2 neutralizing antibodies (also called "anti-spike monoclonal antibodies") do not come from humans. They are made in the lab and work the same way as the antibodies people can develop following an infection with the SARS-CoV-2 virus. Casirivimab + imdevimab (a mixture of two different antibodies) and sotrovimab are examples of SARS-CoV-2 neutralizing antibodies. They can neutralize the SARS-CoV-2 virus by recognizing it, attaching to it, and preventing it from spreading through the body. These monoclonal antibodies are all licensed for use in Canada.

\section{How We Came to Our Recommendations}

There are a variety of randomized clinical trials evaluating different SARS-CoV-2 neutralizing antibodies in different COVID-19 patients: from people who don't have symptoms but had a high-risk exposure to someone infected with COVID-19, to mild illness in people still being cared for home, to people with more severe illness who need to be in the hospital. These trials were conducted at different stages of the pandemic, with different virus variants circulating. Some of the studies took place early in the pandemic, where doctors and nurses didn't have many other drugs to treat COVID-19. We took all of this into consideration. We also considered that Canada does not have a large supply of SARS-CoV-2 neutralizing antibodies right now. We believe most Canadians want to use drugs that will help them in important ways, like preventing to get seriously ill with COVID-19, to end up in the hospital, or preventing to die. We also believe most Canadians think about how treatments for COVID-19 might reduce the burden on our health care system, to ensure as many people as possible get good care - whether they have COVID-19 or not.

Our Recommendations for Moderately and Severely III Patients Who are Sick Enough to be in Hospital

If you are within 9 days of starting to have any symptom of COVID-19, you may benefit from casirivimab + imdevimab. If you have never had COVID-19, are not fully vaccinated (with two doses of Vaxzevria, Comirnaty, and/or Spikevax), and a doctor feels you are rapidly getting sicker, you should get casirivimab + imdevimab $8000 \mathrm{mg}$ IV. You do not need a test to see if you already have your own anti-spike antibodies. If you have never had COVID-19 and are not fully vaccinated (with two doses of Vaxzevria, Comirnaty, and/or Spikevax), but your medical condition is stable, you may get casirivimab + imdevimab 8000 mg IV - but only if an antibody test shows you do not already have your own antibodies. If you do have your own anti-spike antibodies, you should not get SARS-CoV-2 neutralizing antibodies.

If you have had COVID-19, and/or are fully vaccinated (with two doses of Vaxzevria, Comirnaty, and/or Spikevax) but your medical condition is stable, you may get casi- 
rivimab + imdevimab $8000 \mathrm{mg}$ IV - but only if an antibody test shows you do not already have your own anti-spike antibodies. If you do have your own anti-spike antibodies, you should not get SARS-CoV-2 neutralizing antibodies.

\section{Our Recommendations for Mildly III Patients Who are Sick, but Still Well Enough to be Cared for Outside of Hospital}

At this time, we recommend that Ontarians receive casirivimab + imdevimab, not sotrovimab. Casirivimab + imdevimab is probably more convenient to use since it can be given either as an infusion into the veins or as an injection under the skin.

If you are within 7 days of starting to have any symptom of COVID-19, you may benefit from casirivimab + imdevimab. If your symptoms started more than 7 days ago, you should not get it.

People with COVID-19 who are still well enough to be cared for outside of the hospital do not need to be tested to see if they have their own antibodies if they are being considered for SARS-CoV-2 neutralizing antibodies. They do need to have symptoms of COVID-19, and their infection needs to be confirmed with an appropriate lab test.

If you have never had COVID-19 and are not fully vaccinated (with two doses of Vaxzevria, Comirnaty, and/or Spikevax) and have at least one underlying risk factor, you should get casirivimab + imdevimab $1200 \mathrm{mg}$ IV or SC.

If you have had COVID-19 and/or are fully vaccinated (with two doses of Vaxzevria, Comirnaty, and/or Spikevax) and you are immunocompromised or immunosuppressed, you may get casirivimab + imdevimab 1200 mg IV or SC.

If you have had COVID-19 and/or are fully vaccinated (with two doses of Vaxzevria, Comirnaty, and/or Spikevax) and you have no underlying risk factors (including no immunocompromise or immunosuppression), you should not get SARS-CoV-2 neutralizing antibodies.

\section{Background}

The SARS-CoV-2 spike protein is involved in receptor recognition, viral attachment, and entry into host cells. ${ }^{1}$ Neutralizing antibodies work by binding non-competitively to the critical receptor binding domain of SARS-CoV-2's spike protein, thereby stopping the virus from binding to and entering human cells. Passive immunization with antispike neutralizing monoclonal antibodies appears to prevent disease progression in recently diagnosed COVID-19 patients.

Currently there are three SARS-CoV-2 neutralizing antibodies authorized by interim order from Health Canada: bamlanivimab (LY-CoV-555), casirivimab + imdevimab (marketed in the European Union and United Kingdom as Ronapreve, and as REGENCOV in the United States), and sotrovimab (Sotrovimab). Bamlanivimab monotherapy is no longer recommended for use in COVID-19, due to inadequate neutralizing activity against SARS-CoV-2 variants of concern; the combination of bamlanivimab and etesevimab is not currently approved for use in Canada. This brief will address the use of casirivimab (CAS-ih-RIH-vih-mab) + imdevimab (IM-DEH-vih-mab), and sotrovimab (so-TROE-vih-mab); these recombinant human monoclonal antibodies bind to distinct but overlapping regions on the SARS-CoV-2 spike protein thereby preventing viral entry into cells.

Health Canada has recently approved these products for treatment of COVID-19 in mild to moderately ill non-hospitalized patients who are at a high risk for progression to hospitalization and/or death.

Casirivimab + imdevimab is approved as a single intravenous (IV) infusion of $2400 \mathrm{mg}$ (1200 mg of each monoclonal antibody component) over a minimum of 60 minutes, with monitoring for at least 60 minutes after infusion. Sotrovimab is approved as a 
single IV infusion of $500 \mathrm{mg}$ over 60 minutes, with monitoring for 60 minutes after infusion. However, casirivimab + imdevimab has been used at a dose of $8000 \mathrm{mg}$ IV (4000 mg of each monoclonal antibody component) in hospitalized patients, ${ }^{2}$ and has been administered subcutaneously at a dose of $1200 \mathrm{mg}(600 \mathrm{mg}$ of each monoclonal antibody component) in asymptomatic household contacts of COVID-19. ${ }^{3}$

It is expected that the demand for SARS-CoV-2 neutralizing antibodies will be high in the current and future waves of the pandemic; individuals who do not have existing immunity (either because they are unvaccinated, or because they are immunocompromised/ immunosuppressed and cannot mount an adequate immune response to vaccination) are at the highest risk of serious outcomes. Demand for SARSCoV-2 neutralizing monoclonal antibodies will therefore correlate with case counts of non-immune individuals.

There are federal, provincial, and territorial frameworks to manage drug shortages in Canada, enhanced during the COVID-19 pandemic. Furthermore, frameworks exist to manage drug allocation. However, increasing numbers of patients with COVID-19 eligible for treatment with SARS-CoV-2 neutralizing antibodies may pose a challenge for the allocation and distribution of medications that are costly and in limited supply, due to a high demand worldwide.

\section{Questions}

What is the evidence to support use of SARS-CoV-2 neutralizing antibodies in patients with moderate/severe COVID-19?

What is the evidence to support use of SARS-CoV-2 neutralizing antibodies in patients with mild COVID-19?

What is the evidence to support use of SARS-CoV-2 neutralizing antibodies in postexposure prophylaxis settings?

What clinical recommendations has the Clinical Practice Guidelines Working Group (CPG WG) made around the use of SARS-CoV-2 neutralizing antibodies in symptomatic COVID-19?

Are there special considerations with pregnancy and lactation?

What factors must be considered to promote ethical and equitable allocation of SARSCoV-2 neutralizing antibodies?

How can we optimize the distribution, supply, and administration of SARS-CoV-2 neutralizing antibodies to maximize benefit?

\section{Findings}

\section{What Is the Evidence to Support Use of Sars-Cov-2 Neutralizing Antibodies in Patients with Moderate/Severe COVID-19?}

The RECOVERY trial investigated the effect of casirivimab + imdevimab in patients hospitalized with COVID-19. ${ }^{2}$ It randomized patients to one of three arms: usual care and convalescent plasma; usual care and casirivimab + imdevimab; or usual care alone. The convalescent plasma arm was stopped early for lack of benefit. The results of the casirivimab + imdevimab arm of RECOVERY have been published as a preprint.

Eligible patients had clinically suspected or lab confirmed COVID-19, were over age 12 , weighed over $40 \mathrm{~kg}$, and in the judgement of the treating clinician, did not have a contraindication to casirivimab + imdevimab. Previous SARS-CoV-2 infection or vaccination did not affect eligibility. Pregnant and breastfeeding people were eligible for randomization. Between September 2020 and May 2021, 9785 hospitalized patients at 127 sites in the UK randomized to the casirivimab + imdevimab arm received a 
single $8000 \mathrm{mg}$ IV infusion (4000 mg casirivimab/4000 mg imdevimab) infused over $60+/-15$ minutes.

Baseline characteristics were similar between the usual care and the usual care plus casirivimab + imdevimab groups. At randomization, $62 \%$ of the patients required oxygen therapy, $26 \%$ required non-invasive ventilation (with $6 \%$ progressing to invasive ventilation), and $7 \%$ required no oxygen therapy. Nearly all patients enrolled in the trial received steroids (94\%). Fewer received remdesivir (25\%) and tocilizumab (14\%). Baseline antibody testing revealed $54 \%$ of patients had baseline SARS-CoV-2 antibodies (seropositive patients) and 32\% were antibody negative (seronegative patients), with $14 \%$ missing antibody data. However, baseline antibody testing was not used for stratification of randomization. The median time from symptom onset was 9 days for all patients (interquartile range 6 to 12 days), and 7 days for seronegative patients (interquartile range 6 to 10 days).

Among all patients in the trial there was no difference in death at 28 days, discharge alive from hospital, and progression to invasive mechanical ventilation or death. There was no significant harm signal, including infusion-related adverse events.

Among seronegative patients, casirivimab + imdevimab was associated with reduced mortality at 28 days compared to usual care ( $24 \%$ in casirivimab + imdevimab vs $30 \%$ in usual care, RR 0.80 confidence interval $(\mathrm{Cl}) 0.70-0.91$, p-value $=0.001)$, increased probability of discharge alive from hospital (64\% vs 58\%, RR $1.19 \mathrm{Cl} 1.08-1.30$, p < 0.001 ), and reduced progression to invasive mechanical ventilation or death (30\% vs $37 \%$, RR $0.83 \mathrm{Cl} 0.75-0.82, \mathrm{p}<0.001$ ). Casirivimab + imdevimab conferred an absolute risk reduction of 6 fewer deaths at 28 days for 100 patients treated.

Among seropositive patients, there was no difference in nearly all of these outcomes and those with unknown antibody status. However, there was a trend to increased mortality at 28 days in patients who were seropositive and received casirivimab + imdevimab (16\% vs $15 \%$, RR $1.09 \mathrm{Cl} 0.95-1.26)$. Due to the time window of enrollment, it is likely that most enrolled patients in the casirivimab + imdevimab arms of RECOVERY were not infected with the Delta variant.

\section{What Is the Evidence to Support Use of Sars-Cov-2 Neutralizing Antibodies in Patients with Mild COVID-19?}

There are two published trials on the use of casirivimab + imdevimab, and sotrovimab, in patients with mild COVID-19.

\section{Casirivimab + Imdevimab}

Casirivimab + imdevimab was investigated in a multi-center, randomized, doubleblind, placebo-controlled trial of non-hospitalized patients by Weinreich et al. ${ }^{4}$ The results of the phase 3 study, are presented here. ${ }^{4}$

Non-hospitalized laboratory-confirmed COVID-19 patients were originally randomized to receive placebo, casirivimab + imdevimab $2400 \mathrm{mg} \mathrm{IV}$, or casirivimab + imdevimab $8000 \mathrm{mg}$ IV. Based on phase 1 and 2 results that showed these doses of casirivimab + imdevimab had similar clinical efficacy, and that most clinical events occurred in highrisk patients, the trial protocol was amended. Subsequent patients were randomized to placebo, casirivimab + imdevimab $1200 \mathrm{mg}$ IV, or casirivimab + imdevimab 2400 $\mathrm{mg}$ IV; they were also required to have at least one risk factor for severe COVID-19 (i.e., age $>50$, obesity with $\mathrm{BMI}>30$, cardiovascular disease including hypertension, chronic lung disease including asthma, diabetes mellitus, chronic liver disease, chronic kidney disease, or immunocompromised state due to medical condition or immunosuppressive medication).

Eligible patients had laboratory-confirmed COVID-19, onset of any COVID-19 symptoms 
within 7 days of randomization, and were 18 years of age or older. Patients with hypoxia (SpO2 < 93\% on room air) were excluded from the trial. Patients who were pregnant at randomization were enrolled in the trial, but the results of this cohort have not yet been reported. Patients who were previously vaccinated or infected with COVID-19 were not excluded. The primary endpoint was a composite outcome of allcause mortality or COVID-19-related hospitalization at 29 days. Key secondary clinical endpoints were: all-cause mortality or a COVID-19-related hospitalization from day 4 through day 29; and COVID-19 symptom resolution.

Patients were enrolled between September 24, 2020 and January 17, 2021 at 115 sites, mostly in the United States. On February 19, 2021 the trial stopped enrolling patients in the placebo arm. In the amended phase III protocol, 2519 patients with at least one risk factor underwent randomization. 2048 patients with at least one risk factor were also included from the original phase III protocol, which randomized patients to $8000 \mathrm{mg}$ or $2400 \mathrm{mg}$ arms.

Baseline characteristics were similar between the usual care and casirivimab + imdevimab groups. The most common risk factor for severe COVID-19 was obesity (58\%). Baseline antibody testing revealed $23.6 \%$ of patients had baseline SARS-CoV-2 antibodies (seropositive), and $68.6 \%$ were antibody negative (seronegative), with $7.8 \%$ missing antibody data. Baseline antibody testing was not used to determine treatment allocation.

In terms of the primary clinical endpoint, both casirivimab + imdevimab $2400 \mathrm{mg}$ and $1200 \mathrm{mg}$ significantly reduced COVID-19-related hospitalization or all-cause death compared to placebo ( $71.3 \%$ relative risk reduction [ $1.3 \%$ vs $4.6 \% ; p<0.0001$ ] and $70.4 \%$ relative reduction $[1.0 \%$ vs $3.2 \% ; p=0.0024$ ], respectively). Comparable relative risk reductions were achieved by both baseline seronegative and baseline seropositive patients compared to placebo. Similarly, there were comparable relative risk reductions in both the $2400 \mathrm{mg}$ and $1200 \mathrm{mg}$ arms. There was no significant harm signal, including infusion-related adverse events.

Symptom resolution occurred 4 days earlier in both treatment dose groups than in the placebo groups ( 10 days vs 14 days, respectively; $p<0.0001,2400 \mathrm{mg}$ and 1200 $\mathrm{mg})$. Those treated with casirivimab + imdevimab who were hospitalized had nonstatistically significant shorter stays ( $2400 \mathrm{mg}$, median 6.0 vs 7.0 days, $n=80 ; 1200 \mathrm{mg}$, 4.0 vs 5.5 days, $n=31$ ) and were less likely to be admitted to the ICU than those treated with placebo who were hospitalized $(2400 \mathrm{mg}, 0.4 \%$ vs $1.3 \%$, relative risk reduction $67 \%$ [95\% Cl $17.2-86.9] ; 1200 \mathrm{mg}, 0.4 \%$ vs $0.9 \%$, relative risk reduction $56.4 \%[95 \% \mathrm{Cl}$ 67.8-88.7]).

Due to the time window of enrollment, it is likely that most enrolled patients in the trial were not infected with the Delta variant.

\section{Sotrovimab}

Sotrovimab was investigated in a multi-center, randomized, double-blind, placebocontrolled trial of non-hospitalized patients by Gupta et al. ${ }^{5}$ The results of the phase 3 study, currently published as a preprint, are presented here.

Non-hospitalized symptomatic COVID-19 patients were randomized to an IV infusion of sotrovimab $500 \mathrm{mg}$ or placebo $(n=583)$. They were also required to have at least one risk factor for disease progression (i.e., age $\geq 55$, obesity with $\mathrm{BMI}>30$, diabetes mellitus, congestive heart failure New York Heart Association class $>1$, chronic kidney disease GFR $<60 \mathrm{~mL} / \mathrm{min}$, chronic obstructive pulmonary disease, or moderate to severe asthma).

Eligible patients had laboratory-confirmed COVID-19 (positive polymerase chain reaction (PCR) or antigen testing), onset of any COVID-19 symptoms within 5 days 
of randomization, and were 18 years of age or older. Patients were excluded if they had respiratory distress, dyspnea at rest, or requiring supplemental oxygen. Patients who were pregnant or breastfeeding, severely immunocompromised, and those who received a COVID-19 vaccine at any point prior to enrollment were excluded. Patients who had prior infection were not excluded. The primary efficacy endpoint was the proportion of patients with COVID-19 progression, defined as hospitalization longer than 24 hours, or death, through day 29.

Patients were enrolled between August 27, 2020 and January 19, 2021 at 37 sites in the United States, Canada, Brazil, and Spain. Baseline characteristics were similar between the usual care and the sotrovimab groups. The most common risk factors were obesity (63\%), age 55 years or older (47\%), and diabetes requiring medication (23\%). Baseline antibody testing was not reported.

In terms of the primary clinical endpoint, the risk of COVID-19 progression was significantly reduced in the sotrovimab arm (85\% relative risk reduction [1\% vs $7 \%$ ], 97.24\% $\mathrm{Cl}$ 44-96, $\mathrm{p}=0.002$ ); the confidence interval was adjusted for the interim analysis. There was no significant harm signal, including infusion-related adverse events. Due to the time window of enrollment, it is likely that most enrolled patients were not infected with the Delta variant.

\section{What Is the Evidence to Support Use of Sars-Cov-2 Neutralizing Antibodies in Post- exposure Prophylaxis Settings?}

There are two studies that have explored post-exposure prophylaxis in contacts of persons with COVID-19.

The first study, BLAZE-2, ${ }^{6}$ was a multi-center, randomized, double-blind, placebocontrolled trial of residents and staff in long-term care and assisted living facilities in the United States by Cohen et al. The results of the peer-reviewed phase 3 trial are presented here.

Uninfected residents $(n=300)$ and staff $(n=666)$ at a facility with at least 1 confirmed SARS-CoV-2 case were randomized to bamlanivimab $4200 \mathrm{mg}$ or placebo. Eligible subjects had to have negative PCR testing and serology. The primary efficacy endpoint was symptomatic PCR-positive COVID-19 within 8 weeks of randomization.

Patients were enrolled between August 2 and November 20, 2020 at 74 facilities in the United States. Baseline characteristics were similar between the bamlanivimab and placebo arms. In terms of the primary endpoint, the incidence of symptomatic COVID-19 progression was significantly reduced in the bamlanivimab arm $43 \%$ reduction [ $8.5 \%$ vs $15.2 \%$ ], $95 \% \mathrm{Cl} 28-68, \mathrm{p}<0.001$ ). There was no significant harm signal, including infusion-related adverse events. Due to the time window of enrollment, subjects were unvaccinated and not exposed to the Delta variant.

The second trial was a multi-center, randomized, double-blind, placebo-controlled trial of previously uninfected household contacts of infected persons (Part A) and recently infected asymptomatic persons (Part B) by O'Brien et al. ${ }^{3}$ The results of the peer-reviewed phase 3 study of Part $A$ are presented here.

Uninfected participants 12 years of age or older were enrolled within 96 hours after a household contact was diagnosed with SARS-CoV-2 infection to receive casirivimab

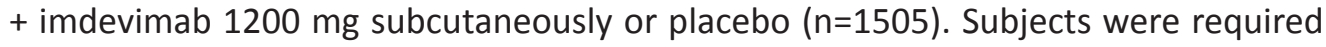
to be RT-qPCR negative for SARS-CoV-2 and previously uninfected, but the primary efficacy analysis consisted of patients who were also seronegative at baseline. The primary efficacy endpoint was symptomatic RT-qPCR positive for SARS-CoV-2 within 28 days of randomization.

Patients were enrolled until January 28, 2021 at 112 sites in the United States, Romania, and Moldova. Baseline characteristics were similar between the casirivimab 
+ imdevimab and placebo arms. In terms of the primary endpoint, the incidence of symptomatic COVID-19 progression was significantly reduced in the bamlanivimab arm ( $81 \%$ relative risk reduction [ $1.5 \%$ vs $7.8 \%$ ],$p<0.001$ ). There was no significant harm signal, although $4.2 \%$ of subjects receiving casirivimab + imdevimab experienced an injection-site reaction compared to $1.5 \%$ receiving placebo. Due to the time window of enrollment, subjects were not exposed to the Delta variant.

As mentioned above, bamlanivimab is authorized by Interim Order through Health Canada. However, it is now accepted that bamlanivimab monotherapy possesses insufficient neutralizing activity against the Delta variant. ${ }^{7}$ Bamlanivimab in combination with etesemivab, and etesemivab alone, may retain sufficient neutralizing activity against the Delta variant, but etesemivab is currently not approved by Health Canada; furthermore, it appears that bamlanivimab in combination with etesevimab has reduced neutralization activity against the Beta, Gamma, and "Delta plus" (AY.1/ AY.2) variants. ${ }^{8,9}$

What Clinical Recommendations Has the CPG WG Made around the Use of SarsCov-2 Neutralizing Antibodies in Symptomatic COVID-19?

\section{Moderately/Critically III Patients}

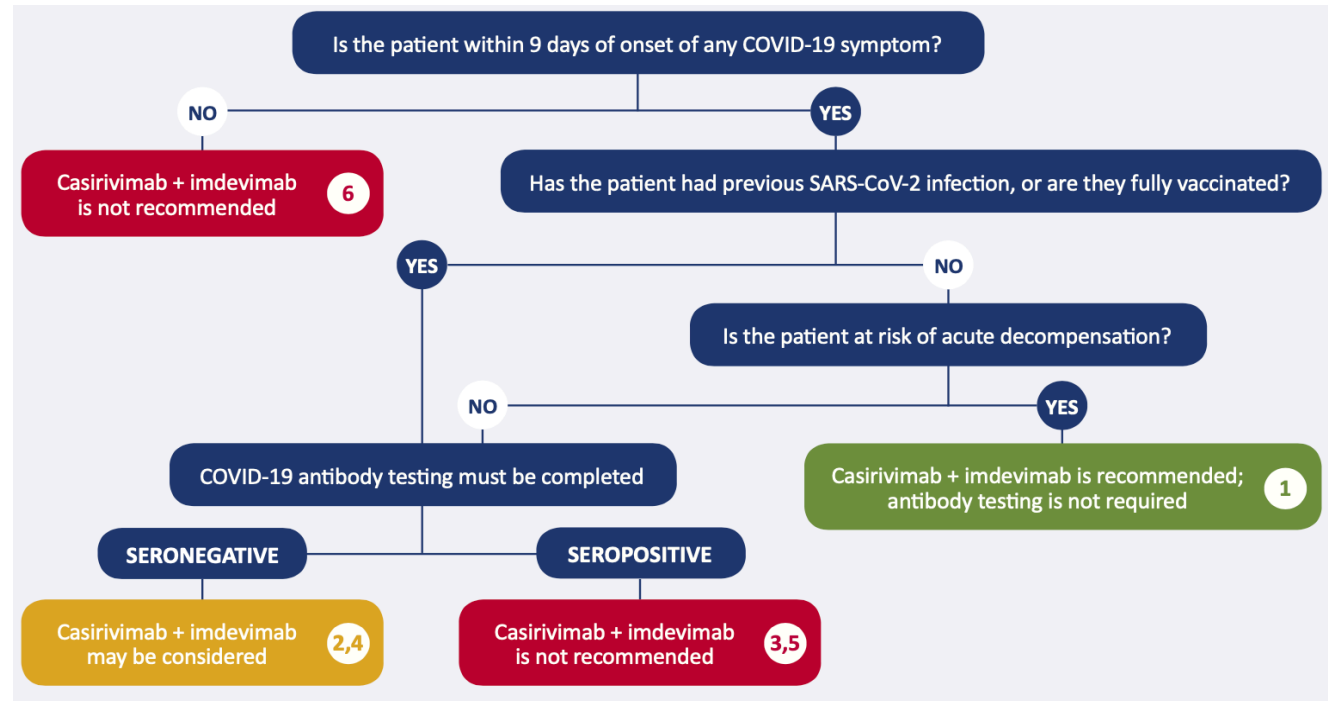

Figure 1. Graphical Summary of Recommendations for Moderately/Critically III Patients

Recommendation 1. Unstable patients with no history of SARS-CoV-2 infection or full vaccination

The monoclonal antibody cocktail casirivimab + imdevimab at a dose of $8000 \mathrm{mg}$ IV is recommended for moderately/critically ill patients with no history of full vaccination or SARS-CoV-2 infection, who are within 9 days of onset of any COVID-19 symptom, and have demonstrated rapid clinical deterioration. Anti-spike antibody testing is not required.

Recommendation 2. Stable patients with no history of SARS-CoV-2 infection or full vaccination, seronegative

The monoclonal antibody cocktail casirivimab + imdevimab at a dose of $8000 \mathrm{mg}$ IV may be considered for moderately/critically ill patients with no history of full vaccination or SARS-CoV-2 infection, who are within 9 days of onset of any COVID-19 symptom, and are not clinically at risk of acute decompensation, if SARS-CoV-2 antispike antibody testing demonstrates they are seronegative.

Recommendation 3. Stable patients with no history of SARS-CoV-2 infection or full vaccination, seropositive 
The monoclonal antibody cocktail casirivimab + imdevimab is not recommended for moderately/critically ill patients with no history of full vaccination or SARS-CoV-2 infection, who are within 9 days of onset of any COVID-19 symptom, and are not clinically at risk of acute decompensation, if SARS-CoV-2 anti-spike antibody testing demonstrates they are seropositive.

Recommendation 4. Patients with a history of SARS-CoV-2 infection or full vaccination, seronegative

The monoclonal antibody cocktail casirivimab + imdevimab at a dose of $8000 \mathrm{mg}$ IV may be considered for moderately/critically ill patients with a history of full vaccination or SARS-CoV-2 infection, who are within 9 days of onset of any COVID-19 symptom, if SARS-CoV-2 anti-spike antibody testing demonstrates they are seronegative.

Recommendation 5. Patients with a history of SARS-CoV-2 infection or full vaccination, seropositive

The monoclonal antibody cocktail casirivimab + imdevimab is not recommended for moderately/critically ill patients with a history of full vaccination or SARS-CoV-2 infection, who are within 9 days of onset of any COVID-19 symptom, if SARS-CoV-2 anti-spike antibody testing demonstrates they are seropositive.

\section{Recommendation 6. Patients beyond 9 days of onset of COVID-19 symptoms}

The monoclonal antibody cocktail casirivimab + imdevimab is not recommended for moderately/critically ill patients who are beyond 9 days of onset of any COVID-19 symptom, whether or not they are presumed to have immunity through previous infection or full vaccination.

\section{Mildly III Patients}

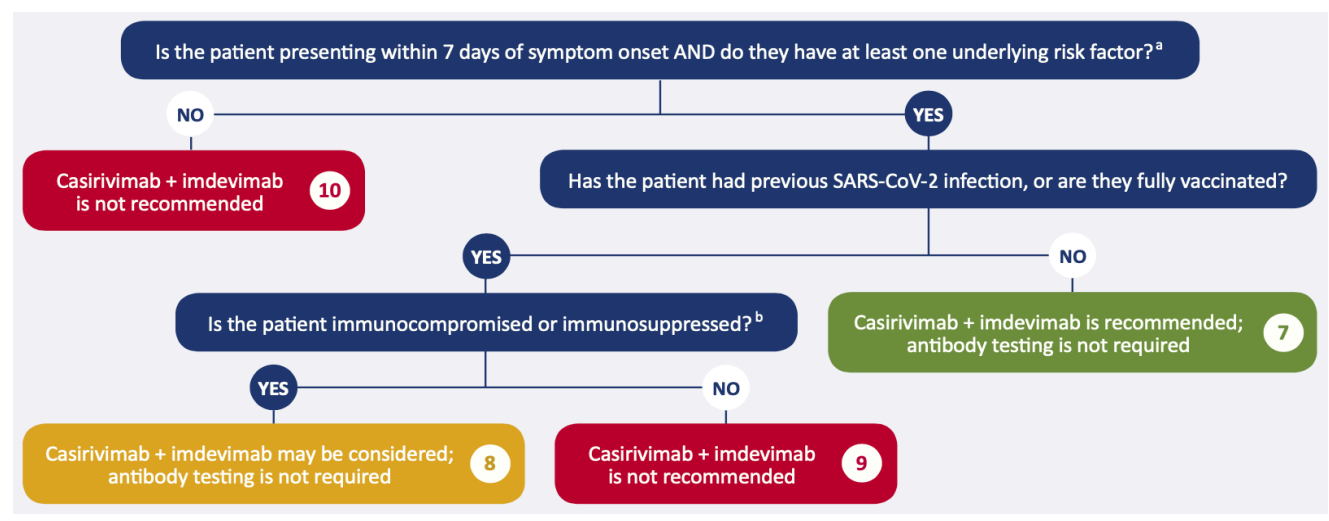

Figure 2. Graphical Summary of Recommendations for Mildly III Patients

a Risk factors: age >50 years, obesity, cardiovascular disease (including hypertension), chronic lung disease (including asthma), chronic metabolic disease (including diabetes), chronic kidney disease, chronic liver disease, immunosuppression $^{b}$, or receipt of immunosuppressants ${ }^{b}$.

${ }^{b}$ Examples include: active treatment for solid tumor and hematologic malignancies, receipt of solid-organ transplant and taking immunosuppressive therapy, receipt of chimeric antigen receptor (CAR)-T-cell or hematopoietic stem cell transplant (within 2 years of transplantation or taking immunosuppression therapy), moderate or severe primary immunodeficiency (e.g., DiGeorge syndrome, Wiskott-Aldrich syndrome), advanced or untreated HIV infection, active treatment with high-dose corticosteroids (i.e., $\geq 20 \mathrm{mg}$ prednisone or equivalent per day when administered for $\geq 2$ weeks), alkylating agents, antimetabolites, transplant-related immunosuppressive drugs, cancer chemotherapeutic agents classified as severely immunosuppressive, tumor-necrosis factor (TNF) blockers, and other biologic agents that are immunosuppressive or immunomodulatory.

Recommendation 7. Patients with no history of SARS-CoV-2 infection or full vaccination, with risk factors

The monoclonal antibody cocktail casirivimab + imdevimab at a dose of $1200 \mathrm{mg}$ IV or $\mathrm{SC}$ is recommended for mildly ill patients who meet the following criteria:

- No history of full vaccination or SARS-CoV-2 infection, AND 
- Confirmed, symptomatic COVID-19, AND

- Within 7 days of onset of any COVID-19 symptom, AND

- At least one of the following risk factors: age > 50, obesity, cardiovascular disease (including hypertension), chronic lung disease (including asthma), chronic metabolic disease (including diabetes), chronic kidney disease, chronic liver disease, immunosuppression, or receipt of immunosuppressants) ${ }^{1}$

- Anti-spike antibody testing is not required

Recommendation 8. Patients with a history of SARS-CoV-2 infection or full vaccination, immunocompromised or immunosuppressed

The monoclonal antibody cocktail casirivimab + imdevimab at a dose of $1200 \mathrm{mg}$ IV or SC may be considered for mildly ill patients who meet the following criteria:

- History of previous SARS-CoV-2 infection or full vaccination, AND

- Confirmed symptomatic COVID-19 AND within 7 days of onset of any COVID-19 symptom, AND

- Immunocompromised or immunosuppressed

- Anti-spike antibody testing is not required

Examples of immunocompromised or immunosuppressed individuals include individuals with active treatment for solid tumor and hematologic malignancies, receipt of solid-organ transplant and taking immunosuppressive therapy, receipt of chimeric antigen receptor (CAR)-T-cell or hematopoietic stem cell transplant (within 2 years of transplantation or taking immunosuppression therapy), moderate or severe primary immunodeficiency (e.g., DiGeorge syndrome, Wiskott-Aldrich syndrome), advanced or untreated HIV infection, active treatment with high-dose corticosteroids (i.e., $\geq 20 \mathrm{mg}$ prednisone or equivalent per day when administered for $\geq 2$ weeks), alkylating agents, antimetabolites, transplant-related immunosuppressive drugs, cancer chemotherapeutic agents classified as severely immunosuppressive, tumornecrosis factor (TNF) blockers, and other biologic agents that are immunosuppressive or immunomodulatory. For individuals who are immunosuppressed or receiving immunosuppressants, their condition is considered both an underlying risk factor AND a marker of insufficient ability to mount an immune response to SARS-CoV-2.

Those with no history of infection or vaccination are covered under non-immune to SARS-CoV-2.

Recommendation 9. Patients with a history of SARS-CoV-2 infection or full vaccination, with risk factors other than immunocompromise or immunosuppression

Monoclonal antibody therapy is not recommended for all other patients with presumed immunity (through full vaccination or previous infection).

Recommendation 10. Patients with a history of SARS-CoV-2 infection or full vaccination, with no risk factors

Monoclonal antibody therapy is not recommended for patients at low risk of adverse outcomes, whether or not they are presumed to have immunity.

Recommendation 11. Use of sotrovimab

At this time, casirivimab + imdevimab is preferred over sotrovimab $500 \mathrm{mg}$ IV due to practical considerations; the former currently has greater availability, and has IV and SC formulations. 


\section{Are There Special Considerations with Pregnancy and Lactation?}

There are currently limited high-quality data on the use of casirivimab + imdevimab and sotrovimab in COVID-19 patients who are pregnant or lactating - a situation which applies to the overall management of COVID-19 in these individuals. However, given the mechanism of action of SARS-CoV-2 neutralizing antibodies, and the wellestablished safety profile of products like IV immunoglobulin and plasma in individuals who are pregnant or lactating, these agents are presumed to be safe. They should be used if the potential benefit justifies the potential risk - as they would in any patient. Compared with non-pregnant reproductive-aged peers diagnosed with COVID-19, the relative rates of hospitalization and admission to ICU were significantly increased (RR 4.26 95\% Cl 3.45-5.10 - hospitalization; RR 11.39 95\% Cl 7.90-15.21 0 ICU admission). ${ }^{10}$ The Working Group reinforced that principles of COVID-19 management that apply to non-pregnant, non-lactating patients should be equitably applied to pregnant or lactating patients as well. Pregnant patients with COVID-19 should ideally be cared for by multidisciplinary teams familiar with the management of pregnancy.

\section{What Factors Must Be Considered to Promote Ethical, Equitable Allocation of Sars- Cov-2 Neutralizing Antibodies?}

There is strong mechanistic and clinical evidence supporting the use of monoclonal antibodies early in SARS-CoV-2 infection, especially in those who do not have preexisting antibodies (i.e., seronegative patients). There are clear barriers to allocating drugs and biologics ethically and equitably, however strategies can be put in place to address these barriers.

During periods of drug scarcity, drug doses are an important consideration. Seronegative patients admitted to hospital with COVID-19 require over six times the dose of casirivimab + imdevimab (8000 mg) compared to outpatients with mild COVID-19 and risk factors for severe disease $(1200 \mathrm{mg})$. They also require baseline antibody testing to confirm their serostatus if they are stable. However, the hospitalized population is logistically easier to identify and treat, as no new infrastructure is required to administer an IV infusion to an inpatient. Laboratory infrastructure is required to provide timely baseline anti-spike antibody testing results. There are a variety of different SARS-CoV-2 serological assays available at present. Assays that do not test for anti-spike protein antibodies (e.g., that test for anti-nucleocapsid antibodies) are not appropriate to determine seronegativity, as they will miss protection conferred by COVID-19 immunization.

Conversely, evidence supports foregoing baseline anti-spike antibody testing in outpatients eligible for casirivimab + imdevimab, and the required dose is far lower (thus far less costly and resource-conserving). Yet, the implementation barriers in this group are more significant. They become more manageable if the route of administration shifts from IV infusion to SC administration. Pharmacokinetic data, virology data, and indirect evidence from a trial of casirivimab + imdevimab in asymptomatic patients with early SARS-CoV-2 infection suggest that SC administration of casirivimab + imdevimab is a reasonable alternative to IV administration; this could address implementation barriers, while still conferring therapeutic benefit for treatment of COVID-19 (note, however, that post-administration monitoring of at least 60 minutes is unchanged between SC and IV dosing). ${ }^{3}$

The median time to reach maximum serum concentration ( $\mathrm{Cmax}$ ) following a single SC $1200 \mathrm{mg}$ dose of casirivimab + imdevimab is $7-8$ days. The observed serum concentrations 1 hour after SC infusion is roughly $27 \%$ that of IV infusion. Serum concentrations 28 days after a single $1200 \mathrm{mg}$ IV or SC dose are comparable. ${ }^{11}$ The Working Group acknowledges that the SC use of casirivimab + imdevimab for COVID-19 treatment is based on indirect data from the SC use of casirivimab + imdevimab for 
post-exposure prophylaxis. ${ }^{3}$ The Group believes this extrapolation of data is justified as casirivimab + imdevimab is rapidly absorbed when administered subcutaneously, the mean concentration of each antibody component (casarivimab, imdevimab) in serum the day after administration is 22.1 and $25.8 \mathrm{mg} / \mathrm{L}$, respectively, and concentrations continue to rise after day 1 through day 7 post-administration. The neutralization dose for casirivimab + imdevimab is approximately $20 \mathrm{mg} / \mathrm{L}$ and does not substantially differ for the Alpha and Delta variants, and the "wild type" SARS-CoV-2., ${ }^{711}$

Based on current clinical evidence, there are two approaches for using casirivimab + imdevimab that will reduce hospital ward and ICU bed use. These two approaches are: 1) treating moderately/critically ill hospitalized, seronegative patients; and 2) treating mildly ill high-risk symptomatic outpatients. It is unclear which approach is more beneficial. For every 1 hospitalized patient treated, 6 high-risk outpatients could be treated. The number of outpatients needed to treat to prevent one hospitalization based on clinical trial data ranges from 17 to 30 . Similarly, the number of hospitalized patients needing to be treated to prevent one ICU admission is 25 , and to prevent one death is 18 , based on clinical trial data.

The Working Group appreciates that the decisions to treat these two populations are not mutually exclusive; treating outpatients will likely reduce the need to treat hospitalized patients. Additionally, recent data from $\mathrm{ClHI}$ shows that patients who would be eligible for outpatient therapy, ${ }^{12}$ when hospitalized, have a length of stay of 20 days, compared with 11 days for those without comorbidities; they also were more likely to require ICU admission ( $28 \%$ vs $18 \%$ ). Treating outpatients may well reduce community spread (including spread to vulnerable community-dwelling individuals who are unvaccinated, or who cannot mount a robust immune response). Treating hospitalized patients may also reduce nosocomial spread (including spread to vulnerable hospitalized patients and to healthcare providers). Any treatment decision will have an impact on health and human resource needs in Ontario.

There are important ethical issues inherent in the implementation of SARS-CoV-2 neutralizing antibody treatment. Based on historic patterns of infection and current immunization rates, the spread of severe COVID-19 during the 4th wave may differ from prior waves, preferentially impacting communities with low immunization rates. This includes rural and remote communities that cannot easily access tertiary hospital care. Any implementation must account for structural urbanism, a bias in health care towards large population centers due to: ${ }^{13}$

- a market orientation in health care which necessitates a critical mass of customers to make services viable;

- a public health focus on changing outcomes at the population level, which differentially allocates funding toward large population centers;

- and the innate inefficiencies of low-population and remote settings, in which even equal funding will not translate into equitable funding.

The risk of structural urbanism may be profound in treatment with monoclonal antibodies, especially for outpatients who require timely diagnosis and then timely access to antibody administration.

\section{How Can We Optimize the Distribution, Supply, and Administration of Sars-Cov-2 Neutralizing Antibodies to Maximize Benefit?}

At the time of publication, Ontario is experiencing the fourth wave due to the Delta variant. Patients at highest risk of poor outcomes are those who are unvaccinated or immunocompromised. SARS-CoV-2 neutralizing antibodies have been shown to save lives, prevent hospitalization, and reduce ICU admission in eligible patients. However, the demand from potentially eligible patients may exceed supply in the coming weeks 
based on projected cases, hospitalizations, and ICU admissions. ${ }^{14}$

Ontario's at-risk population will consist of those who are unvaccinated, and those who fail to mount an effective immune response to vaccination (either from immunosuppression or waning immunity). It is unlikely that policies related to monoclonal antibody eligibility (i.e., widespread availability) will substantially influence vaccine uptake among those currently unvaccinated. Should unvaccinated individuals become infected, they will remain at substantial risk of severe illness, hospitalization, and death from COVID-19. The Working Group emphasized that treatment should not be withheld from eligible patients (in accordance with direction provided herein) solely for punitive reasons (i.e., because these individuals chose not to be vaccinated) or solely for coercive reasons (i.e., to compel these individuals to be vaccinated).

These considerations dictate that it is critical to develop a robust conservation and implementation plan for SARS-CoV-2 neutralizing antibodies in Ontario that ensures eligible patients receive it in an ethical, equitable, evidence-informed fashion.

\section{Operational Considerations}

Operational considerations for efficient use include the optimal use of drug vials. Casirivimab + imdevimab is supplied as casirivimab + imdevimab concentrate for infusion, as either two single-use $6 \mathrm{~mL}$ vials (each containing $300 \mathrm{mg}$ of one monoclonal antibody) or two single-use $20 \mathrm{~mL}$ vials (each containing $1332 \mathrm{mg}$ of one monoclonal antibody) per package. Centers which only have access to $20 \mathrm{~mL}$ vials, for example, would risk wasting product by administering a $1200 \mathrm{mg}$ dose to a single patient. Consideration must be given to distributing the vials in a fashion that would minimize wastage.

Use can also be optimized by using SC injection as an alternative route of administration whenever IV administration poses a barrier, is not feasible, and/or would lead to a delay in treatment. An example would include treatment in the ambulatory setting. Clinical monitoring after injection for at least 1 hour is recommended regardless of the route of administration. Implementation of SARS-CoV-2 neutralizing antibodies, specifically in the outpatient setting, will require creation of accessible, equitable infrastructure with trained staff to administer the drug and supervise patients after infusion.

Choice of agent is another strategy that might influence operational considerations. Currently, the Working Group has made a recommendation for casirivimab + imdevimab over sotrovimab, as the former is more available, and is available for both SC and IV administration. Going forward, decisions on which SARS-CoV-2 neutralizing antibody to use should be based on clinical evidence, as well as local/regional factors that include availability, the need for different formulations, and activity against locally circulating variants.

\section{Applying a Risk Framework to Patient Selection}

SARS-CoV-2 neutralizing antibodies have been shown to reduce hospitalization, ICU admission, and death in outpatients. These neutralizing antibodies have also been shown to reduce ICU admission and death in inpatients. Due to limited supply, consideration can be given to administering this drug to individuals who are most at risk for poor outcomes from COVID-19. ${ }^{15}$ The largest study of casirivimab + imdevimab in outpatients (by Weinreich et al.) relies heavily on risk factors to capture eligible patients; the trial's list of risk factors is very broad, and not all risk factors impact patient outcomes equally. ${ }^{4}$ The following proposed risk framework for SARS-CoV-2 neutralizing antibody use in outpatients is based on current published international and Canadian data around highest risk prognostic factors for mortality as an outcome of COVID-19: ${ }^{16-25}$ 
1. If drug supply is very restricted, eligible outpatients should be: $\geq 70$ years of age; or $\geq 50$ years of age AND First Nations, Inuit, or Metis. ${ }^{26,27}$ All patients should have a reasonable expectation for 1-year survival prior to getting infected with SARS-CoV-2

2. As drug supply expands, eligible outpatients should: have Down Syndrome and be $\geq 18$ years of age; ${ }^{22}$ or be $\geq 60$ years of age AND have at least one additional risk factor; or be $\geq 40$ years of age AND First Nations, Inuit, or Metis AND have at least one additional risk factor. ${ }^{26,27}$ Additional risk factors are as follows:

- Obesity (BMI $\geq 30)$

- Dialysis, or stage 5 kidney disease $($ eGFR < 15)

- Diabetes

- Cerebral palsy

- Intellectual disability (of any severity)

- Sickle cell disease

- Receiving active cancer treatment

- Solid organ or stem cell transplant recipients

All patients should have a reasonable expectation for 1-year survival prior to getting infected with SARS-CoV-2.

3. IIf supply expands further, eligible outpatients should include those in the original recommendations cited above (i.e., age $\geq 50$, obesity, cardiovascular disease (including hypertension), chronic lung disease (including asthma), chronic metabolic disease (including diabetes), chronic kidney disease, chronic liver disease, immunosuppression, or receipt of immunosuppressants). ${ }^{1}$ Consideration could be given to other persons at increased risk, including younger adults with cerebral palsy, intellectual disability, sickle cell disease, and pregnant individuals. These individuals should have a reasonable expectation for 1-year survival prior to getting infected with SARS-CoV-2.

The CPG WG acknowledges the limitations of the above risk framework, given the many factors that impact baseline risk with COVID-19 (some of which likely act synergistically), the challenges of establishing the presence of important baseline risk factors (including frailty, and the chance of 1-year survival prior to SARS-CoV-2 infection), and the uncertainty over whether SARS-CoV-2 neutralizing antibodies have different effects in different clinical subgroups.

\section{Ethics- And Evidence-Based Considerations}

An ethical, evidence-based framework is required to inform allocation and use of limited SARS-CoV-2 neutralizing antibody supply. The Ontario COVID-19 Bioethics Table adapted a published Ontario drug supply shortage framework for use during the COVID-19 pandemic. ${ }^{28,29}$ It outlines a set of guiding ethical principles (Figure 3 ) and three stages for managing drug supply during the COVID-19 pandemic:

Stage 1: Preserve the standard of care for as many COVID-19 patients as possible by conserving supply, sharing supply, and procuring or accessing new supply.

Stage 2: Optimize therapeutic benefit based on existing evidence within available supply (Primary Allocation Principle).

Stage 3: Use a fair procedure to choose between COVID-19 patients (Secondary Allocation Principle) if Stage 1 and 2 efforts are insufficient to meet demand. 


\begin{tabular}{|c|c|}
\hline Principle & Description \\
\hline Beneficence & $\begin{array}{l}\text { Maintain highest quality of safe and effective care based on available evidence and } \\
\text { within available resources (i.e. drug supply) }\end{array}$ \\
\hline Equity & Ensure fair and non-discriminatory access to resources \\
\hline Reciprocity & Respond to each other in similar ways that recognize mutual interdependence \\
\hline Solidarity & $\begin{array}{l}\text { Build, preserve and strengthen inter-professional / -institutional / -sectoral / } \\
\text {-provincial collaborations and partnerships }\end{array}$ \\
\hline Stewardship & $\begin{array}{l}\text { Use available resources responsibly (e.g., to optimize therapeutic benefit and } \\
\text { sustain available supply) }\end{array}$ \\
\hline Trust & $\begin{array}{l}\text { Foster and maintain public, patient and healthcare provider confidence in health } \\
\text { system }\end{array}$ \\
\hline Utility & $\begin{array}{l}\text { Maximize the greatest possible good for the greatest possible number of individuals } \\
\text { with available resources }\end{array}$ \\
\hline
\end{tabular}

Figure 3. Guiding Ethical Principles for Managing Drug Shortages ${ }^{29}$

Guided by these ethical principles, a set of recommendations was developed for allocation of tocilizumab in COVID-19's 3rd wave. The Working Group believes these recommendations should be adopted to rationally allocate SARS-CoV-2 neutralizing antibody at each stage of the Ontario drug supply shortage framework.

\section{Recommendations and Options}

If Ontario were to enter a SARS-CoV-2 neutralizing antibody shortage, which it may based on the estimated number of eligible patients, and the known current supply of drug available through the Public Health Agency of Canada - it would need to consider procurement of additional product, and approaches that optimize the effectiveness and equity of the existing supply. Working from the Ontario COVID-19 Bioethics Table framework noted above, this Science Brief provides recommendations and options at each stage.

\section{All Stages of Managing Casirivimab + Imdevimab Supply and Distribution}

- Use should follow the Ontario COVID-19 Science Advisory Table's Clinical Practice Guidelines, which are supported by randomized, controlled clinical trials (supported by beneficence). The Ontario COVID-19 Science Advisory Table's Recommendations account for the best available evidence in identifying COVID-19 patients with proven benefit from casirivimab + imdevimab therapy.

- Casirivimab + imdevimab should be allocated province-wide to optimize therapeutic benefits and ensure equitable access for all eligible COVID-19 patients in Ontario (supported by beneficence, equity, reciprocity, solidarity, and trust). Particular attention should be paid to where populations most likely to benefit are currently located. This would include proactively understanding where the highest concentrations of unvaccinated individuals reside and seek care, and the disease epidemiology in these locations. Centralized or coordinated allocation at the provincial and/or regional need may at least partially mitigate structural urbanism.

- The minimum effective dose of casirivimab + imdevimab should be used in line with the evidence in this brief (supported by beneficence, equity, stewardship, and utility). As mentioned above, there are also pharmacologic and clinical rationales for using SC administration in the outpatient setting. These two strategies would immediately increase supply for eligible Ontario COVID-19 patients.

\section{Options for All Stages of Managing Drug Supply and Distribution}

- The assessment of the stage of antibody shortage should be based on predicted demand and communicated clearly to all clinical stakeholders (supported by beneficence, equity, and stewardship). Exponential growth of SARS-CoV-2 and 
changing public health measures in the community makes it difficult for most people to predict future SARS-CoV-2 neutralizing antibody needs. This requires mathematical modeling support to estimate the total future needs based on expected supply. If only ICU admissions are available, a reasonable estimate of all hospitalized COVID-19 patients (ICU and non-ICU) can be acquired by increasing this threefold (in the RECOVERY trial, the ratio of patients mechanically ventilated to those non-mechanically ventilated or without ventilatory support was approximately 1:1). ${ }^{30}$ Using this figure, and similar Ontario-specific information on cases, hospitalizations, and ICU admissions, a projection of demand in subsequent weeks can be made, with re-evaluation (and possibly re-calibration) weekly depending on observed usage.

- Ontario could create a near real-time provincial dashboard accessible to all clinical stakeholders that includes currently available casirivimab + imdevimab supply, and allocated and administered doses (by region, site, and indication). This dashboard could also include any anticipated shipments (supported by beneficence, equity, reciprocity, solidarity, and trust). Having transparent data creates trust and a sense of solidarity, as all participants share with the need to preserve resources. Additionally, it makes it easier to recognize where some regions or sites might have substantially depleted supply, facilitating quicker responsiveness. This principle of a near-real time dashboard was why Ontario's Critical Care Information System (CCIS) was created: in 2003, Severe Acute Respiratory Syndrome (SARS) highlighted the need to improve Ontario's critical care system. In 2006, Ontario's Ministry of Health and Long-Term Care (now separated into MOH and MLTC) announced Ontario's Critical Care Strategy, and CCIS data collection began in 2007. CCIS data has been available throughout the COVID-19 pandemic to support monitoring and managing ICU bed and ventilator capacity.

- There could be a provincially coordinated mechanism building on the Incident Management Systems (IMS) to allow transfer of medication (or, rarely, patients) to ensure that any eligible COVID-19 patient can receive timely, evidence-based therapy (supported by equity and solidarity). Because of the sparse supply and cost of casirivimab + imdevimab and the uneven distribution of COVID-19 patients across the province, patients may present to health care facilities that will not have access to rapid baseline serologic testing and/or casirivimab + imdevimab.

- The optimal turnaround time (TAT) from laboratory receipt for serologic testing is 24 hours, however laboratory resource constraints, transport time, and availability of testing on the weekends in different parts of the province may make it challenging to meet this TAT and ensure clinicians have rapid access to test results on which to base their clinical decisions. Regions are encouraged to establish processes with their referral laboratory (which in some cases, is the Public Health Ontario Laboratory) that optimize pre-analytical factors and set expectations around TAT.

\section{Recommendations for Stage $\mathbf{2}$ of Casirivimab + Imdevimab Supply and Distribution}

- Triaging COVID-19 inpatients for casirivimab + imdevimab therapy based on additional clinical factors deemed to be associated with additional benefit from therapy is not advisable: the data are insufficient to define groups of COVID-19 patients most likely to benefit from treatment. Denying COVID-19 patients with milder illness might increase the number of patients requiring mechanical ventilation. Operationally, this adds challenges that are unlikely to result in a consistent and equitable allocation of casirivimab + imdevimab.

\section{Options for Stage $\mathbf{2}$ of Casirivimab + Imdevimab Supply and Distribution}

- Below, we outline issues around inpatient supply. However, outpatient supply adds an additional complexity - especially recognizing that in the event of a 
shortage, decisions will need to be made between giving $8000 \mathrm{mg}$ of casirivimab

+ imdevimab to 1 inpatient vs. $1200 \mathrm{mg}$ to roughly 6 outpatients.

- Distribution of product could be weekly and site-specific, with allocation based on prior week's consumption (supported by equity, stewardship, and trust). With rapid changes in patient numbers, and potential redistribution of COVID-19 patients using an IMS, a prudent approach to distribution would be weekly distribution of antibodies to regions according to an IMS command structure. The IMS command structure can then distribute further based on local needs and supplies.

- Critical care triage guidelines are applicable to surges that occur in a short period of time, and allocation demands immediate choice. Additionally, ventilators cannot be saved today for use tomorrow. In Ontario, these triage guidelines (i.e., the Ontario Critical Care COVID-19 Command Centre's Emergency Standard of Care) cannot be applied unilaterally by a hospital and can only be applied at the direction of the IMS (i.e., Ontario's Critical Care COVID-19 Command Centre). A similar process is not applicable to the case of SARS-CoV-2 neutralizing antibodies as planning for antibody shortages allows allocation in a manner that has greater equity.

\section{Recommendations for Stage 3 of Managing Casirivimab + Imdevimab Shortages}

- Develop a logic-based mechanism to fairly ration casirivimab + imdevimab (supported by beneficence, equity, solidarity, stewardship, trust, and utility). If Stage 1 and 2 efforts are insufficient to meet demand, Ontario will need to proceed to Stage 3, and use a fair procedure to choose between COVID-19 patients of equal need for secondary prioritization of casirivimab + imdevimab. ${ }^{28,31}$ Stage 3 rationing should not occur on a site-by-site basis but provincially, ideally managed through an IMS.

- SARS-CoV-2 neutralizing antibodies should be given to COVID-19 patients with a reasonable chance of benefiting from it. Because of casirivimab + imdevimab scarcity, it is defensible not to offer these agents to COVID-19 patients who almost certainly will not benefit. This should be based on consensus among those involved in assessing eligibility for allocation.

- A strictly first-come, first-served approach is not recommended, as it would favour patients presenting to centres with known SARS-CoV-2 neutralizing antibody supply and would certainly reinforce structural urbanism and other health inequities by benefiting those in urban centres (with existing or ready supply) over rural locations.

- Casirivimab + imdevimab administration should continue to be guided by supporting evidence, and COVID-19 patients who meet the eligibility criteria but do not want active medical treatment or specific treatment with monoclonal antibody therapy should neither be offered it nor entered into an allocation system.

\section{Options for Stage 3 of Casirivimab + Imdevimab Supply and Distribution}

- To mitigate potential bias influencing the assessment of COVID-19 patients eligible for casirivimab + imdevimab, a second-opinion or consensus model among those responsible for assessing eligibility should be considered.

- An allocation lottery could be the fair default procedure for choosing among eligible COVID-19 patients where allocation based on maximizing benefits cannot be determined. Allocation lotteries have been successfully used in several North American jurisdictions and acknowledges that the efforts to allocate based on maximizing the benefits of therapy have been exhausted. An added potential benefit of an allocation lottery framework is that it could track outcomes of 
COVID-19 patients offered or declined access to therapy. This would allow for evaluation of real-world use in Ontario. We have described potential allocation lotteries in previous Briefs. ${ }^{29}$

\section{Interpretation}

There is mechanistic and clinical evidence to support the use of SARS-CoV-2 neutralizing antibodies in select ambulatory and hospitalized patients with symptomatic COVID-19. Provincial demand for these agents may exceed supply in the near future. A scienceand ethics-guided approach designed to maximize the benefits of available supply is recommended.

Core recommendations include a focus on ethics and equity in the use of these agents; optimizing the dose and administration of these agents; adhering to evidence-based use; establishing a provincial dashboard of supply and distribution accessible to all stakeholders; and ongoing modeling to estimate supply-to-demand adequacy.

\section{Methods Used for This Science Brief}

We searched PubMed and Google Scholar using the following search terms: "sotrovimab," "REGEN-COV, "bamlanivimab," "casirivimab," and "imdevimab." In addition, we retrieved reports citing relevant articles through Google Scholar and reviewed references from identified articles for additional studies. The search was last updated on September 29, 2021. Expert perspectives were sought from critical care medicine, medical ethics, internal medicine, and pharmacy in Ontario, and we also contacted experts from the United States (JAJ and EKM) who had designed and/ or implemented state-wide drug randomization during the COVID-19 pandemic, to provide us with detailed information on various issues around implementation of weighted and unweighted drug lottery systems.

For therapeutic recommendations, we used the following definitions for COVID-19 disease severity:

\section{Critically / Severely III}

Patients requiring ventilatory and/or circulatory support, including high-flow nasal oxygen, non-invasive ventilation, invasive mechanical ventilation, or extracorporeal membrane oxygenation (ECMO). These patients are usually managed in an intensive care setting.

\section{Moderately III}

Patients newly requiring low-flow supplemental oxygen. These patients are usually managed in hospital wards.

\section{Mildly III}

Patients who do not require new or additional supplemental oxygen from their baseline status, IV fluids, or other physiological support. These patients are usually managed in an ambulatory/outpatient setting.

\section{Author Contributions}

JB, AMM and MP conceived the Science Brief. JB wrote the first draft of the Science Brief. All authors revised the Science Brief critically for important intellectual content and approved the final version.

\section{References}

1. Taylor PC, Adams AC, Hufford MM, de la Torre I, Winthrop K, Gottlieb RL. 
Neutralizing monoclonal antibodies for treatment of COVID-19. Nat Rev Immunol. 2021;21(6):382-393. https://doi.org/10.1038/s41577-021-00542-x

2. Horby PW, Mafham M, Peto L, et al. Casirivimab and imdevimab in patients admitted to hospital with COVID-19 (RECOVERY): A randomised, controlled, open-label, platform trial.; 2021:2021.06.15.21258542. https://doi. org/10.1101/2021.06.15.21258542

3. O'Brien MP, Forleo-Neto E, Musser BJ, et al. Subcutaneous REGEN-COV antibody combination to prevent Covid-19. N Engl J Med. Published online August 4, 2021. https://doi.org/10.1056/NEJMoa2109682

4. Weinreich DM, Sivapalasingam S, Norton T, et al. REGEN-COV antibody combination and outcomes in outpatients with Covid-19. N Engl J Med. Published online September 29, 2021. https://doi.org/10.1056/NEJMoa2108163

5. Gupta A, Gonzalez-Rojas Y, Juarez E, et al. Early Covid-19 treatment with SARSCoV-2 neutralizing antibody sotrovimab. medRxiv. Published online May 28, 2021. https://doi.org/10.1101/2021.05.27.21257096

6. Cohen MS, Nirula A, Mulligan MJ, et al. Effect of bamlanivimab vs placebo on incidence of COVID-19 among residents and staff of skilled nursing and assisted living facilities: A randomized clinical trial. JAMA. 2021;326(1):46-55. https://doi. org/10.1001/jama.2021.8828

7. Planas D, Veyer D, Baidaliuk A, et al. Reduced sensitivity of SARS-CoV-2 variant Delta to antibody neutralization. Nature. 2021;596(7871):276-280. https://doi. org/10.1038/s41586-021-03777-9

8. The U.S Food and Drug Administration (FDA). Fact sheet for health care providers Emergency Use Authorization (EUA) of bamlanivimab and etesevimab.; 2021:43. https://www.fda.gov/media/145802/download

9. Public Health Emergency. Resumption in use and distribution of bamlanivimab/ etesevimab in all U.S. states, territories, and jurisdictions. Published September 2, 2021. https://www.phe.gov/emergency/events/COVID19/ investigation-MCM/Bamlanivimab-etesevimab/Pages/resume-distributionbamlanivimabetesevimab-all-states-2sept2021.aspx

10. Munshi L, Wright JK, Zipursky J, et al. The incidence, severity, and management of COVID-19 in critically ill pregnant individuals. Science Briefs of the Ontario COVID-19 Science Advisory Table. 2021;2(43). https://doi.org/10.47326/ ocsat.2021.02.43.1.0

11. The U.S Food and Drug Administration (FDA). Fact sheet for health care providers Emergency Use Authorization (EUA) of REGEN-COV (casirivimab and imdevimab).; 2021. https://www.fda.gov/media/145611/download

12. Canadian Institute for Health Information. COVID-19 hospitalization and emergency department statistics. Published August 26, 2021. https://www.cihi. ca/en/covid-19-hospitalization-and-emergency-department-statistics

13. Probst J, Eberth JM, Crouch E. Structural urbanism contributes to poorer health outcomes for rural America. Health Aff (Millwood). 2019;38(12):1976-1984. https://doi.org/10.1377/hlthaff.2019.00914

14. Ontario COVID-19 Science Advisory Table. Update on COVID-19 Projections.; Science Briefs of the Ontario COVID-19 Science Advisory Table. 2021. https:// covid19-sciencetable.ca/sciencebrief/update-on-covid-19-projections-10/

15. Hunter C. Manitoba First Nations COVID-19 bulletin's. Assembly of Manitoba Chiefs. Published September 20, 2021. https://manitobachiefs.com/covid-19- 
updates-and-information/

16. Izcovich A, Ragusa MA, Tortosa F, et al. Prognostic factors for severity and mortality in patients infected with COVID-19: A systematic review. PloS One. 2020;15(11). https://doi.org/10.1371/journal.pone.0241955

17. Gates M, Pillay J, Wingert A, et al. Risk factors associated with severe outcomes of COVID-19: An updated rapid review to inform national guidance on vaccine prioritization in Canada. Published online May 28, 2021:2021.04.23.21256014. https://doi.org/10.1101/2021.04.23.21256014

18. Bennett TD, Moffitt RA, Hajagos JG, et al. Clinical characterization and prediction of clinical severity of SARS-CoV-2 infection among US adults using data from the US national COVID cohort collaborative. JAMA Netw Open. 2021;4(7):e2116901. https://doi.org/10.1001/jamanetworkopen.2021.16901

19. Ioannou GN, Locke E, Green P, et al. Risk factors for hospitalization, mechanical ventilation, or death among 10131 US veterans with SARS-CoV-2 infection. JAMA Netw Open. 2020;3(9). https://doi.org/10.1001/jamanetworkopen.2020.22310

20. Jehi L, Ji X, Milinovich $A$, et al. Development and validation of a model for individualized prediction of hospitalization risk in 4,536 patients with COVID-19. PLoS One. 2020;15(8):e0237419. https://doi.org/10.1371/journal.pone.0237419

21. Clift AK, Coupland CAC, Keogh RH, Hemingway H, Hippisley-Cox J. COVID-19 mortality risk in Down syndrome: Results from a cohort study of 8 million adults. Ann Intern Med. 2021;174(4):572-576. https://doi.org/10.7326/M20-4986

22. Lunsky $Y$, Durbin A, Balogh R, Lin E, Palma L, Plumptre L. COVID-19 positivity rates, hospitalizations and mortality of adults with and without intellectual and developmental disabilities in Ontario, Canada. Disabil Health J. Published online July 26, 2021:101174. https://doi.org/10.1016/j.dhjo.2021.101174

23. Hüls $A$, Costa $A C S$, Dierssen $M$, et al. Medical vulnerability of individuals with Down syndrome to severe COVID-19-data from the Trisomy 21 Research Society and the UK ISARIC4C survey. EClinicalMedicine. 2021;33:100769. https://doi. org/10.1016/j.eclinm.2021.100769

24. Williamson EJ, McDonald HI, Bhaskaran K, et al. Risks of COVID-19 hospital admission and death for people with learning disability: Population based cohort study using the OpenSAFELY platform. BMJ. 2021;374. https://doi.org/10.1136/ bmj.n1592

25. Hippisley-Cox J, Coupland CA, Mehta N, et al. Risk prediction of COVID-19 related death and hospital admission in adults after covid-19 vaccination: National prospective cohort study. BMJ. 2021;374. https://doi.org/10.1136/bmj.n2244

26. Statistics Canada. First Nations people, Métis and Inuit and COVID-19: Health and social characteristics. Government of Canada. Published April 17, 2020. https:// www150.statcan.gc.ca/n1/daily-quotidien/200417/dq200417b-eng.htm

27. Bresee LC, Knudtson ML, Zhang J, et al. Likelihood of coronary angiography among First Nations patients with acute myocardial infarction. CMAJ. 2014;186(10):E372-E380. https://doi.org/10.1503/cmaj.131667

28. Gibson JL, Bean S, Chidwick P, Godkin D, Wagner RWS and F. Ethical framework for resource allocation during a drug supply shortage. Healthc Q. 2012;15(3). https://www.longwoods.com/content/23040//ethical-framework-for-resourceallocation-during-a-drug-supply-shortage

29. Morris AM, Bean S, Bell CM, et al. Strategies to manage tocilizumab shortages during the COVID-19 pandemic. Science Briefs of the Ontario COVID-19 Science 
Advisory Table. 2021;2(22). https://doi.org/10.47326/ocsat.2021.02.22.1.0

30. Horby PW, Pessoa-Amorim G, Peto L, et al. Tocilizumab in patients admitted to hospital with COVID-19 (RECOVERY): Preliminary results of a randomised, controlled, open-label, platform trial. medRxiv. Published online February 11, 2021:2021.02.11.21249258. https://doi.org/10.1101/2021.02.11.21249258

31. Emanuel EJ, Persad G, Upshur R, et al. Fair allocation of scarce medical resources in the time of Covid-19. N Engl J Med. 2020;382(21):2049-2055. https://doi. org/10.1056/NEJMsb2005114 